\title{
Yield Potential Analysis of Kabuli Chickpea Genotypes at the Limited Water Conditions along with Surveying of the Drought Tolerance Indices
}

\author{
Alireza Taleei ${ }^{* 1}$ and Jalal Shaabani ${ }^{2}$ \\ ${ }^{1}$ Professor in the Department of Agronomy \& Plant breeding, Faculty of \\ Agricultural Sciences and Engineering, College of Agriculture and Natural \\ Resources, University of Tehran, Karaj, P.O. Box 31587-71787, Iran \\ ${ }^{2}$ Post graduate student in the Department of Agronomy \& Plant breeding, Faculty \\ of Agricultural Sciences and Engineering, College of Agriculture and Natural \\ Resources, University of Tehran, Karaj, P. O. Box: 31587-71787, Iran. \\ ataleei@ut.ac.ir,jshaabani@ut.ac.ir
}

\begin{abstract}
Drought mediated-reduce performance is a big challenge for plant breeder in the world; hereupon attempt is characterization and found the response of plant to the stress at the cellular or/and phenotypic levels. Have a classified collection for each crop in inherent properties is a critical necessity for achieving to these goals. Twenty-eight Kabuli lines with two cultivars namely Jam and Kourosh as check varieties have been surveying to screen drought tolerant genotypes in the field. Some of tolerance indices with phenological characters and components of yield considered as measures for the screening. Total dry matter has a robust positive correlation with grain yield. This character followed by 100-seed weight known as the most momentous components in first factor; however, time to maturity and seed numbers were important traits in the second ones by factor analysis. Drought resistance index (DI) detected as key index to distinguish tolerant genotypes of the crop. The genotypes 21, 25, and 166 detected as moer tolerant in the average of drought stress and non-stress conditions. The susceptible genotypes also were 314 and 333 that can were useful bases as extreme selected lines for mechanisms studies in development breeding programs to drought tolerance in chickpea.
\end{abstract}

Keywords: Cicer ariethinum, screening tolerant genotypes, cluster analysis, yield

\section{Introduction}

Chickpea is one of the most important among legumes, which grown in arid and semiarid zones. Due to its long taproot, it can tolerate water deficiency. This plant produced on 700,000 hectares in Iran and ranks fourth in the world after India, Turkey, and Pakistan [1]. Most of chickpea output achieved in the marginal regions at the spring, and due to lack of rainfall during flowering, podding and seed filling times, terminal drought stress is a major environmental stress, which reduce chickpea production in Iran [2]. One of the most important objectives for breeding to drought stress is selection for drought tolerant genotypes of chickpea [3]. The identification of strategies to improvement crop productivity under limited water conditions is a big challenge for plant scientists. The regions of arid and semi-arid in the world are already facing torrid shortage of water; therefore, they subjected at a great risk [4].

Plant breeders have found that achieving a genetically increase performance under stress conditions is a problematic task, while development in yield potential has been much higher in non-stressed conditions [5-6]. The main challenge for breeders is reduction of yield under drought stress conditions and they have used different procedures 
to evaluate genetic differences in drought tolerant genotypes. Hence, drought resistance indices can used for screening tolerant genotypes, which provide a measure of drought tolerance based on loss of yield under stress conditions [7]. It seems that, the performance of genotypes in both drought and non-stress environments is a good starting point for identification of traits related to drought resistance and the selection of genotypes for breeding programs [8].

Evaluating genotypes in resistance to drought can done through multiple indices, which could be proposed using mathematical relationships between stress and non-stress conditions. Drought resistance defined by Hall [9] as the relative yield of a genotype subjected to the same drought stress compared to the other genotypes. Susceptibility to drought often measured as a function of reduction in yield of a genotype under drought stress, whereas the values are confounded with differential yield potential of genotypes [5]. Multiple selection indices have suggested for select the tolerant genotypes based on their yields in stress and non-stress conditions. Fischer et al., [10] proposed that relative drought index (RDI) could be a useful index for indicating stress tolerance. Jusheng [11] defined an indicator for drought resistance index (DI), which commonly accepted to identify genotypes with high yield under both stress and without stress conditions. Rosielle and Hamblin [12] described a stress tolerance index (TOL) as the differences in amount of yield between drought and irrigated environments and mean productivity (MP) as the average yield of genotypes under both stress and non-stress conditions. Because drought stress can vary in severity at the field environments over years, breeders interested in crops relative performance often use the geometric mean productivity (GMP) [13]. One of the most important tolerance indices is stress tolerance index (STI) that defined by Fernandez [14] as a useful tool for determining high yield and stress tolerance potential of genotypes. This study carried out to screen drought tolerance criteria/indices and selection of drought tolerant landraces in some of Kabuli chickpeas of Iran.

\section{Materials and Methods}

This work performed in the research field of the Department of Agronomy and Plant breeding, University College of Agriculture and Natural Resources, University of TehranKaraj, Iran (with latitude $35^{\circ} 56^{\prime} \mathrm{N}$ and longitude $50^{\circ} 58^{\prime} \mathrm{E}$ and altitude of $1112.5 \mathrm{~m}$ above sea level) between February and August 2014. The average annual rainfall based on data of 48 years average is $268 \mathrm{~mm}$ and the amount of rainfall for the research period was 94.5 $\mathrm{mm}$.

\subsection{Plant Materials and Experimental Design}

Twenty-eight chickpea lines selected from departmental gene bank along with two cultivars namely Jam (998) and Kourosh (999) as controls shown in Table 1. A nested completely randomized block design with two replications used to implement the experiment. Each block considered as an environment and all of the genotypes randomly allocated in each block, in a way that two environments contain drought stress and nonstress conditions. The seeds of each line sown in rows with 1-meter length and between row's distance of $0.5 \mathrm{~m}$ and that of between plants were $10 \mathrm{~cm}$. The experiment consists of four blocks, two for drought, and two for non-stress conditions.

\subsection{Data Collection}

Days to $50 \%$ flowering (FL), 50\% podding (PO) and 50\% maturity (MA), as phenological traits recorded for every row during developmental stages of plants. Considering the marginal effect, equal numbers of plants for each line harvested. The rest of traits measured after harvesting of plants including yield (YI), total dry matter (TDM), 100-seed weight (SW) and harvest index (HI). These traits measured by an electronic 
weighing scale. In addition, number of seeds (NS) also recorded. Drought stress applied in 50\% flowering time for all the blocks and since then irrigation terminated in stress condition, however, in non-stress condition continued and it was due to common irrigation regime of the region.

Table 1. The Chickpea Lines Evaluated for Drought Tolerance

\begin{tabular}{cc}
\hline Lines No. & Origin \\
\hline 15 & Iran \\
21 & Iran \\
25 & Iran \\
92 & Iran \\
101 & Iran \\
160 & Iran \\
166 & Iran \\
176 & Iran \\
192 & Iran \\
205 & Iran \\
211 & Iran \\
226 & Iran \\
227 & Iran \\
233 & Iran \\
240 & Iran \\
263 & Iran \\
302 & Iran \\
308 & Iran \\
311 & Iran \\
314 & Iran \\
315 & Iran \\
316 & Iran \\
327 & Iran \\
333 & Iran \\
339 & Iran \\
349 & Iran \\
371 & Iran \\
376 & Iran \\
$998($ Jam) & Iran \\
999 (Kourosh) & Iran \\
\hline &
\end{tabular}

The tolerance indices such as mean productivity (MP), tolerance index (TOL), geometric mean productivity (GMP), stress tolerance index (STI), harmonic mean (HARM), relative drought index (RDI), and drought resistance index (DI) obtained using the following formulas:

$$
\begin{gathered}
\mathrm{MP}=(\mathrm{Yp}+\mathrm{Ys}) / 2 \\
\mathrm{TOL}=\mathrm{Yp}-\mathrm{Ys} \\
\mathrm{GMP}=\sqrt{\mathrm{Yp}+\mathrm{Ys}_{\mathrm{s}}} \\
\mathrm{STI}=(\mathrm{Yp} \times \mathrm{Ys}) /[\mathrm{Yp}] 2 \\
\mathrm{HARM}=[2(\mathrm{Yp} \times \mathrm{Ys})] /(\mathrm{Yp}+\mathrm{Ys}) \\
\left.\mathrm{RDI}=(\mathrm{Ys} / \mathrm{Yp}) / \overline{\mathrm{Y}}_{\mathrm{S}} / \overline{\mathrm{Y}}_{\mathrm{p}}\right) \\
\mathrm{DI}=[\mathrm{Ys} \times(\mathrm{Ys} / \mathrm{Yp})] / \overline{\mathrm{Y}}_{\mathrm{S}}
\end{gathered}
$$


Where, Ys and Yp represent yield in stress and non-stress conditions, respectively, while $\overline{\mathrm{Y}}_{\mathrm{S}}$ and $\overline{\mathrm{Y}}_{\mathrm{p}}$ are mean yield of all genotypes in order for stress and non-stress conditions, too.

\subsection{Analysis}

The average of two replications used to eliminate the probable errors of analyses. Oneway analysis of variance applied for scored traits. Besides, the above-mentioned indices calculated for stress and non-stress conditions, respectively. The genotypes mean yield of each environment compared. In addition, multivariate analyses carried out for the traits and tolerance indices. The obtained data subjected to analysis of variance (ANOVA) with the Statistical Software Package (SAS, version 9.3, SAS Institute Inc. Cary, NC, USA). Factor analysis, principal component analysis, cluster analysis, and biplot analysis as multivariate techniques performed using Statgraphics X64 (Statgraphics Centurion XV1.11, StatPoint Technologies, USA).

\section{Results}

\subsection{Phenological Characters}

Days to $50 \%$ flowering affected by drought stress $(P \leq 0.01)$, and its range varied from $64.25 \pm 0.2$ to $75.25 \pm 1.2$ days for genotype 376 that followed by Jam cultivar, and genotype 316 which followed by genotypes 15, 240, 263, 315, 333 and 349, respectively. Results for podding time showed that the genotype $376(71.00 \pm 0.7)$ followed by Jam cultivar and genotype $316(80.25 \pm 1.4)$ followed by genotypes 333, 211 and 263 have lowest and highest times to rise of pod $(P \leq 0.01)$. High significant effect of drought stress observed on times to maturity $(P \leq 0.01)$. As the significant variation $(P \leq 0.01)$ seen between genotypes in the environment. Whereas minimum of days to maturity observed for genotype $376(97.25 \pm 1.5)$, the maximum of time to maturity belonged to genotype 205 by $109.00 \pm 5.4$ days followed by genotypes 15 and 240 (Table 2 ).

\subsection{Total Dry Matter and Harvest Index}

According to Table 2, it is clear that for total dry matter, the effect of drought stress and genotypes were highly significant and significant, respectively. The highest of total dry matter detected for genotype 21 with $29.52 \pm 5.1 \mathrm{gr}$ plant ${ }^{-1}$, and genotype 314 with7.92 $\pm 0.6 \mathrm{gr}$ plant $^{-1}$ has lowest has lowest amount of within the rest of genotypes. Genotype 176 has the high harvest index of $(0.49 \pm 0.0)$, while that of genotype $333(0.29 \pm 0.0)$ was the lowest one (Table 2).

\subsection{Yield and Its Components}

Grain yield and number of seed were affected by drought stress $(P \leq 0.01)$, whereas the effect of one on 100-seed weight was not significant. The genotypes were different for 100 -seed weight $(P \leq 0.01)$. The largest number of seeds and maximum of grain yield found for genotype 21 with $50.35 \pm 14.9$ seeds plant $^{-1}$ followed genotype 302 , and (11.60 $\pm 3.3 \mathrm{gr} \mathrm{plant}^{-1}$ ) in 21 followed by genotype 101, respectively. The lowest seed numbers $\left(16.90 \pm 1.4\right.$ plant $\left.^{-1}\right)$ and yield $\left(2.32 \pm 0.3\right.$ gr plant $\left.^{-1}\right)$ also observed in genotype 314 followed by genotypes 371 and 333, respectively. Jam cultivar has the high 100-seed weight $\left(26.83 \pm 0.9\right.$ gr plant $\left.^{-1}\right)$ followed by genotypes 101, 166, 192, 371 and 999 (Kourosh) and the lowest amount belongd to genotype 333 followed by genotypes 263, 314, 315, 316, and 327 (Table 2). 


\subsection{Tolerance Indices}

The high values of Ys, MP, GMP, and HARM indices belonged for genotype 21, while those of STI, RDI, and DI values seen for genotype 25. Genotype 101 also has high values for Yp and TOL indexes. However, the smull values of Yp, MP, GMP, and HARM indices belonged for genotype 314. In addition, genotype 339 has the smull values of STI and RDI. The low values of Ys and DI as well as TOL, observed in genotypes 315 and 25, respectively (Table 3 ).

Table 2. Comparison of Trait Means for Genotypes per Single Plant Level in Stress and Non-Stress Conditions

\begin{tabular}{|c|c|c|c|c|c|c|c|c|}
\hline \multirow[t]{2}{*}{ Genotype } & \multicolumn{8}{|c|}{ Traits } \\
\hline & $\begin{array}{c}\text { FL } \\
\text { (days) }\end{array}$ & $\begin{array}{c}\mathrm{PO} \\
\text { (days) }\end{array}$ & $\begin{array}{l}\text { MA } \\
\text { (days) }\end{array}$ & $\begin{array}{c}\text { TDM } \\
\left(\text { gr plant }^{-1}\right)\end{array}$ & $\begin{array}{l}\text { NS } \\
\left(\text { plant }^{-1}\right)\end{array}$ & $\begin{array}{c}\text { YI } \\
\left(\text { gr plant }^{-1}\right)\end{array}$ & $\begin{array}{c}\text { SW } \\
\left(\text { gr plant }^{-1}\right)\end{array}$ & $\begin{array}{l}\mathrm{HI} \\
(\%)\end{array}$ \\
\hline 15 & $74.25 \pm 1.4$ & $77.75 \pm 1.0$ & $108.25 \pm 4.9$ & $22.46 \pm 2.6$ & $32.25 \pm 7.3$ & $7.47 \pm 1.0$ & $21.98 \pm 2.8$ & $0.30 \pm 0.0$ \\
\hline 21 & $71.50 \pm 1.3$ & $75.75 \pm 0.8$ & $105.50 \pm 2.8$ & $29.52 \pm 5.1$ & $50.35 \pm 14.9$ & $11.60 \pm 3.3$ & $23.07 \pm 0.5$ & $0.39 \pm 0.0$ \\
\hline 25 & $69.50 \pm 0.6$ & $74.75 \pm 0.7$ & $103.50 \pm 0.6$ & $21.72 \pm 3.1$ & $37.44 \pm 8.2$ & $8.93 \pm 1.8$ & $24.11 \pm 1.1$ & $0.40 \pm 0.0$ \\
\hline 92 & $69.00 \pm 1.6$ & $74.00 \pm 1.4$ & $101.50 \pm 0.8$ & $17.72 \pm 4.3$ & $28.55 \pm 7.3$ & $6.75 \pm 1.8$ & $23.56 \pm 0.9$ & $0.37 \pm 0.0$ \\
\hline 101 & $70.25 \pm 0.8$ & $74.75 \pm 0.2$ & $104.00 \pm 1.0$ & $24.88 \pm 8.5$ & $44.13 \pm 18.2$ & $11.36 \pm 5.4$ & $24.44 \pm 2.0$ & $0.41 \pm 0.0$ \\
\hline 160 & $68.75 \pm 0.8$ & $74.25 \pm 0.4$ & $102.50 \pm 0.6$ & $15.17 \pm 4.2$ & $36.03 \pm 10.7$ & $6.34 \pm 1.9$ & $20.13 \pm 2.7$ & $0.47 \pm 0.0$ \\
\hline 166 & $69.00 \pm 0.7$ & $74.00 \pm 0.4$ & $105.50 \pm 1.5$ & $19.94 \pm 2.9$ & $31.88 \pm 4.0$ & $8.58 \pm 1.1$ & $26.92 \pm 0.6$ & $0.43 \pm 0.0$ \\
\hline 176 & $67.00 \pm 1.0$ & $74.00 \pm 0.5$ & $102.50 \pm 3.2$ & $17.47 \pm 3.9$ & $42.35 \pm 11.4$ & $8.84 \pm 2.7$ & $20.79 \pm 2.0$ & $0.49 \pm 0.0$ \\
\hline 192 & $73.00 \pm 1.0$ & $77.50 \pm 0.8$ & $105.50 \pm 1.7$ & $20.33 \pm 4.8$ & $33.72 \pm 9.7$ & $8.37 \pm 2.3$ & $24.96 \pm 1.0$ & $0.39 \pm 0.0$ \\
\hline 205 & $72.00 \pm 1.9$ & $77.75 \pm 2.3$ & $109.00 \pm 5.4$ & $18.90 \pm 4.2$ & $29.97 \pm 6.9$ & $6.94 \pm 1.8$ & $23.02 \pm 1.4$ & $0.36 \pm 0.0$ \\
\hline 211 & $72.50 \pm 2.0$ & $79.00 \pm 3.3$ & $107.25 \pm 4.0$ & $17.65 \pm 3.8$ & $39.22 \pm 7.8$ & $7.55 \pm 1.9$ & $18.72 \pm 1.4$ & $0.42 \pm 0.0$ \\
\hline 226 & $70.50 \pm 0.8$ & $75.00 \pm 1.0$ & $105.25 \pm 1.0$ & $17.00 \pm 4.3$ & $34.43 \pm 8.7$ & $8.01 \pm 2.1$ & $20.71 \pm 2.1$ & $0.41 \pm 0.0$ \\
\hline 227 & $70.00 \pm 1.7$ & $75.00 \pm 2.0$ & $103.50 \pm 1.8$ & $14.43 \pm 2.1$ & $27.74 \pm 3.6$ & $6.00 \pm 0.8$ & $21.51 \pm 0.2$ & $0.41 \pm 0.0$ \\
\hline 233 & $71.50 \pm 0.8$ & $74.75 \pm 0.7$ & $104.50 \pm 1.2$ & $13.19 \pm 2.5$ & $25.82 \pm 5.0$ & $4.99 \pm 1.1$ & $16.86 \pm 2.0$ & $0.33 \pm 0.0$ \\
\hline 240 & $74.25 \pm 1.4$ & $77.75 \pm 1.1$ & $108.25 \pm 4.0$ & $11.16 \pm 3.0$ & $25.75 \pm 10.4$ & $4.08 \pm 1.6$ & $15.91 \pm 0.1$ & $0.32 \pm 0.0$ \\
\hline 263 & $74.75 \pm 1.4$ & $79.75 \pm 1.7$ & $104.50 \pm 2.0$ & $11.73 \pm 2.9$ & $26.96 \pm 6.5$ & $4.46 \pm 1.1$ & $14.98 \pm 1.4$ & $0.33 \pm 0.0$ \\
\hline 302 & $71.00 \pm 1.6$ & $74.75 \pm 1.1$ & $104.25 \pm 1.7$ & $19.80 \pm 4.6$ & $47.51 \pm 12.8$ & $9.28 \pm 2.7$ & $18.88 \pm 1.1$ & $0.44 \pm 0.0$ \\
\hline 308 & $70.50 \pm 2.0$ & $74.50 \pm 0.8$ & $104.50 \pm 2.0$ & $22.92 \pm 5.2$ & $41.04 \pm 9.4$ & $9.38 \pm 2.3$ & $22.47 \pm 0.8$ & $0.40 \pm 0.0$ \\
\hline 311 & $71.75 \pm 1.0$ & $74.00 \pm 0.7$ & $101.75 \pm 2.3$ & $14.06 \pm 4.1$ & $37.26 \pm 15.5$ & $6.27 \pm 2.7$ & $16.46 \pm 0.5$ & $0.41 \pm 0.0$ \\
\hline 314 & $72.25 \pm 1.1$ & $76.75 \pm 0.4$ & $101.75 \pm 3.2$ & $7.92 \pm 0.6$ & $16.90 \pm 1.4$ & $2.32 \pm 0.3$ & $13.58 \pm 0.7$ & $0.30 \pm 0.0$ \\
\hline 315 & $73.75 \pm 0.6$ & $78.25 \pm 1.3$ & $104.75 \pm 4.1$ & $11.55 \pm 4.7$ & $32.47 \pm 14.5$ & $4.68 \pm 2.1$ & $14.57 \pm 0.8$ & $0.38 \pm 0.0$ \\
\hline 316 & $75.25 \pm 1.2$ & $80.25 \pm 1.4$ & $106.75 \pm 4.4$ & $13.95 \pm 4.5$ & $41.28 \pm 14.7$ & $5.68 \pm 2.0$ & $13.74 \pm 0.5$ & $0.39 \pm 0.0$ \\
\hline 327 & $72.25 \pm 1.7$ & $74.75 \pm 0.9$ & $102.50 \pm 1.9$ & $12.25 \pm 2.3$ & $29.38 \pm 12.1$ & $4.49 \pm 1.9$ & $14.51 \pm 1.3$ & $0.32 \pm 0.0$ \\
\hline 333 & $75.00 \pm 1.0$ & $80.00 \pm 0.4$ & $106.50 \pm 3.3$ & $11.05 \pm 2.9$ & $25.11 \pm 7.1$ & $3.32 \pm 1.0$ & $12.84 \pm 0.8$ & $0.29 \pm 0.0$ \\
\hline 339 & $70.25 \pm 1.3$ & $73.75 \pm 0.8$ & $103.50 \pm 1.5$ & $18.70 \pm 6.0$ & $34.48 \pm 17.7$ & $7.42 \pm 4.0$ & $20.77 \pm 0.9$ & $0.34 \pm 0.0$ \\
\hline 349 & $74.50 \pm 0.2$ & $77.75 \pm 0.8$ & $105.50 \pm 1.5$ & $15.48 \pm 3.6$ & $32.78 \pm 11.8$ & $5.39 \pm 1.9$ & $16.34 \pm 0.8$ & $0.33 \pm 0.0$ \\
\hline 371 & $67.75 \pm 1.2$ & $72.75 \pm 0.6$ & $102.50 \pm 1.9$ & $15.21 \pm 2.7$ & $19.88 \pm 7.4$ & $5.30 \pm 2.0$ & $26.72 \pm 1.1$ & $0.31 \pm 0.0$ \\
\hline 376 & $64.25 \pm 0.2$ & $71.00 \pm 0.7$ & $97.25 \pm 1.5$ & $13.99 \pm 1.6$ & $26.14 \pm 4.3$ & $5.51 \pm 0.9$ & $21.02 \pm 1.8$ & $0.39 \pm 0.0$ \\
\hline 998 & $65.00 \pm 0.7$ & $71.25 \pm 0.2$ & $103.75 \pm 1.4$ & $23.20 \pm 6.7$ & $37.18 \pm 11.5$ & $9.70 \pm 2.8$ & $26.83 \pm 0.9$ & $0.42 \pm 0.0$ \\
\hline 999 & $67.25 \pm 0.4$ & $72.75 \pm 0.7$ & $102.75 \pm 1.2$ & $15.53 \pm 2.7$ & $24.39 \pm 3.6$ & $6.26 \pm 0.8$ & $25.89 \pm 0.7$ & $0.40 \pm 0.0$ \\
\hline Environment & ${ }^{* * *}$ & $* *$ & ** & *** & ** & *** & ns & $\mathrm{ns}$ \\
\hline $\begin{array}{c}\text { Genotype/ } \\
\text { (Envieonment) }\end{array}$ & $* *$ & ** & $\mathrm{ns}$ & $*$ & ns & ns & $* *$ & ns \\
\hline LSD (5\%) & 2.00 & 1.47 & 1.710 & 2.96 & 5.54 & 1.45 & 2.39 & 0.03 \\
\hline $\mathrm{CV}(\%)$ & 0.776 & 0.595 & 0.911 & 13.679 & 15.572 & 32.914 & 14.073 & 28.012 \\
\hline
\end{tabular}

Each value is the means of four replicates \pm standard error (SE). Fisher protected LSD at $\mathrm{P} \leq 0.05$. FL: days to $50 \%$ of flowering, $\mathrm{PO}$ : days to $50 \%$ of podding, MA: days to $50 \%$ of maturity, TDM: total dry matter, YI: grain yield, NS: number of seed, SW: 100-seed weight, HI: harvest index.

**Significant at $1 \%$ level, *: significant at 5\% level ns: non-significant, CV: Coefficient of Variation. 


\subsection{Partial Correlations}

\subsubsection{Non-Stress Condition}

The correlation between flowering and podding time was highly significant. The total dry matter has high significant correlation with seed numbers, 100-seed weight and significant correlation with yield, respectively, while between total dry matter and harvest index was high negative significant. However, harvest index showed highly significant positive correlation with seed number, yield, and 100-seed weight. The negative significant one observed between 100-seed weight and seed numbers (Table 4).

\subsubsection{Stress Condition}

The correlation between days to flowering and podding was highly significant. The total dry matter has significant one with seed numbers, yield and 100-seed weight, however, that of between total dry matter and harvest index was negatively high significant. Harvest index also showed significant correlation with seed number and 100seed weight, and that with yield was highly significant. The negative correlation was highly significant between 100-seed weight and seed numbers as well (Table 5).

\subsubsection{Stress and Non-Stress Conditions Mean}

The flowering and podding time have positive significant correlation. The correlations between total dry matter and seed numbers, yield, and 100-seed weight were highly significant, while the correlation between total dry matter and harvest index was highly significant and negative, however, those between harvest index showed, seed number and 100 -seed weight were highly significant. The correlation between 100 -seed weight and seed number was high significant and negative (Table 6).

Table 3. Calculated Indices of Drought Tolerance for Genotypes per Single Plant

\begin{tabular}{|c|c|c|c|c|c|c|c|c|c|}
\hline $\begin{array}{c}\text { Genotype } \\
\text { No. }\end{array}$ & $Y p$ & Ys & MP & TOL & GMP & STI & HARM & RDI & DI \\
\hline 15 & 8.467 & 6.489 & 7.478 & 1.978 & 3.867 & 0.766 & 7.347 & 1.559 & 1.102 \\
\hline 21 & 13.486 & 9.725 & 11.605 & 3.762 & 4.818 & 0.721 & 11.301 & 1.467 & 1.554 \\
\hline 25 & 8.949 & 8.917 & 8.933 & 0.033 & 4.227 & 0.996 & 8.933 & 2.027 & 1.968 \\
\hline 92 & 8.890 & 4.630 & 6.760 & 4.260 & 3.677 & 0.521 & 6.088 & 1.059 & 0.534 \\
\hline 101 & 17.233 & 5.488 & 11.361 & 11.745 & 4.767 & 0.318 & 8.325 & 0.648 & 0.387 \\
\hline 160 & 9.293 & 3.390 & 6.341 & 5.903 & 3.561 & 0.365 & 4.967 & 0.742 & 0.274 \\
\hline 166 & 9.004 & 8.160 & 8.582 & 0.844 & 4.143 & 0.906 & 8.561 & 1.844 & 1.639 \\
\hline 176 & 13.499 & 4.188 & 8.843 & 9.311 & 4.205 & 0.310 & 6.392 & 0.631 & 0.288 \\
\hline 192 & 12.428 & 4.321 & 8.374 & 8.107 & 4.093 & 0.348 & 6.412 & 0.707 & 0.333 \\
\hline 205 & 10.113 & 3.777 & 6.945 & 6.336 & 3.727 & 0.374 & 5.500 & 0.760 & 0.313 \\
\hline 211 & 10.791 & 4.316 & 7.554 & 6.474 & 3.887 & 0.400 & 6.166 & 0.814 & 0.383 \\
\hline 226 & 11.199 & 4.839 & 8.019 & 6.360 & 4.005 & 0.432 & 6.758 & 0.879 & 0.463 \\
\hline 227 & 6.592 & 5.410 & 6.001 & 1.182 & 3.464 & 0.821 & 5.943 & 1.670 & 0.984 \\
\hline 233 & 6.379 & 3.617 & 4.998 & 2.762 & 3.162 & 0.567 & 4.616 & 1.153 & 0.454 \\
\hline 240 & 5.300 & 2.866 & 4.083 & 2.434 & 2.858 & 0.541 & 3.720 & 1.100 & 0.343 \\
\hline 263 & 5.077 & 3.863 & 4.470 & 1.214 & 2.990 & 0.761 & 4.387 & 1.548 & 0.651 \\
\hline 302 & 13.718 & 4.843 & 9.281 & 8.875 & 4.308 & 0.353 & 7.159 & 0.718 & 0.379 \\
\hline 308 & 13.007 & 5.765 & 9.386 & 7.243 & 4.333 & 0.443 & 7.989 & 0.902 & 0.566 \\
\hline 311 & 9.309 & 3.242 & 6.276 & 6.067 & 3.543 & 0.348 & 4.809 & 0.709 & 0.250 \\
\hline 314 & 2.461 & 2.190 & 2.326 & 0.271 & 2.157 & 0.890 & 2.318 & 1.810 & 0.432 \\
\hline 315 & 7.333 & 2.037 & 4.685 & 5.296 & 3.061 & 0.278 & 3.188 & 0.565 & 0.125 \\
\hline 316 & 8.400 & 2.973 & 5.686 & 5.427 & 3.372 & 0.354 & 4.392 & 0.720 & 0.233 \\
\hline 327 & 5.769 & 3.228 & 4.498 & 2.542 & 2.999 & 0.559 & 4.139 & 1.138 & 0.400 \\
\hline 333 & 3.663 & 2.979 & 3.321 & 0.684 & 2.577 & 0.813 & 3.286 & 1.655 & 0.537 \\
\hline 339 & 11.722 & 3.137 & 7.429 & 8.585 & 3.855 & 0.268 & 4.949 & 0.544 & 0.186 \\
\hline 349 & 7.837 & 2.960 & 5.399 & 4.877 & 3.286 & 0.378 & 4.297 & 0.768 & 0.248 \\
\hline 371 & 7.020 & 3.598 & 5.309 & 3.422 & 3.258 & 0.513 & 4.757 & 1.043 & 0.409 \\
\hline 376 & 6.683 & 4.343 & 5.513 & 2.340 & 3.321 & 0.650 & 5.265 & 1.322 & 0.625 \\
\hline 998 & 14.091 & 5.311 & 9.701 & 8.780 & 4.405 & 0.377 & 7.714 & 0.767 & 0.443 \\
\hline 999 & 7.736 & 4.800 & 6.268 & 2.936 & 3.541 & 0.620 & 5.924 & 1.262 & 0.660 \\
\hline
\end{tabular}


Where, Yp: grain yield in non-stress condition, Ys: grain yield in stress condition, MP: mean productivity, TOL: tolerance index, GMP: geometric mean productivity, STI: stress tolerance index, HARM: harmonic mean, RDI: relative drought index, DI: drought resistance index.

Table 4. Partial Correlation Coefficients between Traits at Non-Stress Condition

\begin{tabular}{|c|c|c|c|c|c|c|c|}
\hline & Flowering & Podding & Maturity & $\begin{array}{c}\text { Total dry } \\
\text { matter }\end{array}$ & Seed No. & Yield & $\begin{array}{c}\text { 100-seed } \\
\text { weight }\end{array}$ \\
\hline Podding & $0.744^{* *}$ & & & & & & \\
\hline Maturity & $0.117^{\mathrm{ns}}$ & $0.352^{\mathrm{ns}}$ & & & & & \\
\hline Total dry matter & $-0.083^{\mathrm{ns}}$ & $0.148^{\mathrm{ns}}$ & $-0.072^{\mathrm{ns}}$ & & & & \\
\hline Seed No. & $0.204^{\mathrm{ns}}$ & $-0.217^{\mathrm{ns}}$ & $0.162^{\mathrm{ns}}$ & $0.738^{* *}$ & & & \\
\hline Yield & $-0.096^{\mathrm{ns}}$ & $0.028^{\mathrm{ns}}$ & $-0.059^{\mathrm{ns}}$ & $0.506^{*}$ & $0.169^{\mathrm{ns}}$ & & \\
\hline 100 -seed weight & $0.031^{\mathrm{ns}}$ & $-0.211^{\mathrm{ns}}$ & $0.254^{\mathrm{ns}}$ & $0.804^{* *}$ & $-0.909^{* *}$ & $0.016^{\mathrm{ns}}$ & \\
\hline Harvest index & $-0.252^{\mathrm{ns}}$ & $0.280^{\mathrm{ns}}$ & $-0.165^{\mathrm{ns}}$ & $-0.880^{* *}$ & $0.868^{* *}$ & $0.852^{* *}$ & $0.850^{* *}$ \\
\hline
\end{tabular}

**: significant at $1 \%$ level, *: significant at $5 \%$ level, ns: non-significant

Table 5. Partial Correlation Coefficients between Traits for Stress Condition

\begin{tabular}{|c|c|c|c|c|c|c|c|}
\hline & $\begin{array}{c}\text { Flowerin } \\
\mathrm{g}\end{array}$ & Podding & $\begin{array}{c}\text { Maturit } \\
\mathrm{y}\end{array}$ & $\begin{array}{c}\text { Total dry } \\
\text { matter }\end{array}$ & $\begin{array}{l}\text { Seed } \\
\text { No. }\end{array}$ & Yield & $\begin{array}{c}100- \\
\text { seed } \\
\text { weight }\end{array}$ \\
\hline Podding & $0.771^{* *}$ & & & & & & \\
\hline Maturity & $0.235^{\mathrm{ns}}$ & $0.189^{\mathrm{ns}}$ & & & & & \\
\hline $\begin{array}{l}\text { Total dry } \\
\text { matter }\end{array}$ & $0.048^{\mathrm{ns}}$ & $-0.116^{\mathrm{ns}}$ & $-0.068^{\mathrm{ns}}$ & & & & \\
\hline Seed No. & $0.019^{\mathrm{ns}}$ & $-0.064^{\mathrm{ns}}$ & $0.285^{\mathrm{ns}}$ & $0.616^{* *}$ & & & \\
\hline Yield & $-0.045^{\mathrm{ns}}$ & $0.203^{\mathrm{ns}}$ & $-0.239^{\mathrm{ns}}$ & $0.520^{* *}$ & $0.342^{\mathrm{ns}}$ & & \\
\hline $\begin{array}{l}\text { 100-seed } \\
\text { weight }\end{array}$ & $-0.122^{\mathrm{ns}}$ & $-0.054^{\mathrm{ns}}$ & $0.381^{\mathrm{ns}}$ & $0.606^{* *}$ & $0.949^{* *}$ & $\underset{\mathrm{ns}}{0.325}$ & \\
\hline $\begin{array}{l}\text { Harvest } \\
\text { index }\end{array}$ & $0.021^{\mathrm{ns}}$ & $-0.158^{\mathrm{ns}}$ & $0.076^{\mathrm{ns}}$ & $-0.909^{* *}$ & $0.450^{*}$ & $\underset{* *}{0.623}$ & $0.436^{*}$ \\
\hline
\end{tabular}

**: significant at $1 \%$ level, *: significant at $5 \%$ level, ns: non-significant

Table 6. Partial Correlation Coefficients between Traits at Stress and NonStress Condition's Mean

\begin{tabular}{|c|c|c|c|c|c|c|c|}
\hline & Flowering & Podding & Maturity & $\begin{array}{c}\text { Total dry } \\
\text { matter }\end{array}$ & $\begin{array}{l}\text { Seed } \\
\text { No. }\end{array}$ & Yield & $\begin{array}{c}\text { 100-seed } \\
\text { weight }\end{array}$ \\
\hline Podding & $0.628^{* *}$ & & & & & & \\
\hline Maturity & $0.350^{\mathrm{ns}}$ & $0.335^{\mathrm{ns}}$ & & & & & \\
\hline $\begin{array}{l}\text { Total dry } \\
\text { matter }\end{array}$ & $-0.072^{\mathrm{ns}}$ & $0.106^{\mathrm{ns}}$ & $0.044^{\mathrm{ns}}$ & & & & \\
\hline Seed No. & $0.234^{\mathrm{ns}}$ & $\stackrel{-}{-}^{\mathrm{ns}}$ & $0.022^{\mathrm{ns}}$ & $0.514^{* *}$ & & & \\
\hline Yield & $-0.050^{\mathrm{ns}}$ & $0.010^{\mathrm{ns}}$ & $-0.036^{\mathrm{ns}}$ & $0.554^{* *}$ & $0.385^{\mathrm{ns}}$ & & \\
\hline $\begin{array}{c}100 \text {-seed } \\
\text { weight }\end{array}$ & $-0.087^{\mathrm{ns}}$ & $\overline{-}^{-}$ & $0.235^{\mathrm{ns}}$ & $0.588^{* *}$ & $-0.853^{* *}$ & $0.258^{\mathrm{ns}}$ & \\
\hline $\begin{array}{l}\text { Harvest } \\
\text { index }\end{array}$ & $-0.360^{\mathrm{ns}}$ & $0.229^{\mathrm{ns}}$ & $0.048^{\mathrm{ns}}$ & $-0.783^{* *}$ & $0.717^{* *}$ & $0.240^{\mathrm{ns}}$ & $0.599^{* *}$ \\
\hline
\end{tabular}

**: significant at $1 \%$ level, *: significant at $5 \%$ level, ns: non-significant 


\subsection{Cluster Analysis}

\subsection{Characters}

Ward's method used to clustering of genotypes as shown in Figure 1. Four clusters obtained which the first group consists of genotypes 240, 327, 314, 349, 315, 233, 263, and 333, and those of the second one were genotypes 15, 205, 316, and 211. The third and fourth groups included genotypes 92, 371, 376, and cultivar 999, cultivar 998 and genotypes $21,25,311,302,226,176,101,308,166,192,227,339$, and 160, respectively.

\subsubsection{Indices}

The results of cluster analysis for tolerance indices showed in Figure 2. In this case, also four clusters achieved. The first group consists of cultivar 998, and genotypes 101, $176,211,339,226,192,308$, and 302, those of second one were genotypes 160, 311, 205, 315,316 , and 349. The third and fourth groups included genotypes 15, 333, 314, 227, 92, $263,327,240,376,233,371$, and cultivar 999 and genotypes 25, 21, and 166, respectively.

\section{7. Factor Analysis}

\subsubsection{Characters}

Using factor analysis, two factors obtained, which justified $80.90 \%$ of total variation. The first factor with $51.26 \%$ amount of variation has large negative coefficients for flowering and podding, while it has larg positive coefficients for yield, 100-seed weight, total dry matter, harvest index, and number of seed. The second factor explained by, number of seed, days to maturity, flowering, and podding (Tables 7 and 8).

\subsubsection{Indices}

Results of factor analysis showed that $98.44 \%$ from variations were justified by two factors. GMP, MP, Yp, HARM, TOL, and Ys indices tolerant have larg positive coefficients for first factor, while for second, TOL has large negative coefficient and DI, STI, RDI and Ys have larg positive coefficients (Table 9 and 10).

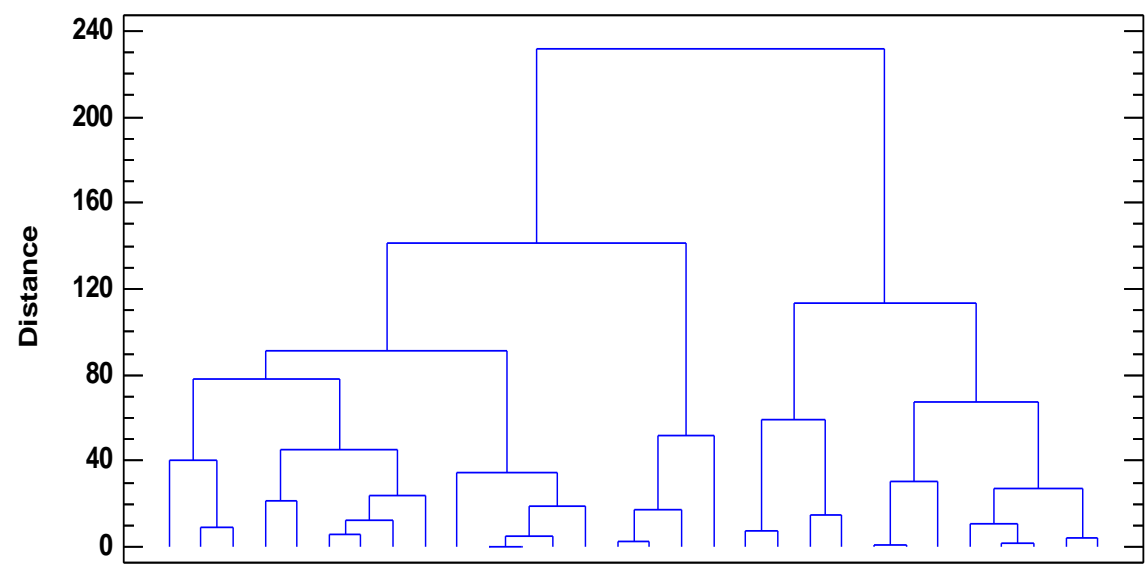

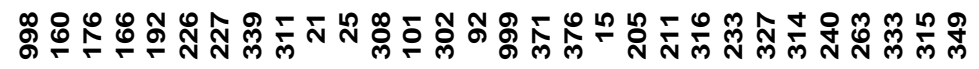

Figure 1. Dendrogram of Mean Genotypes Using Stress and Non-Stress Conditions 


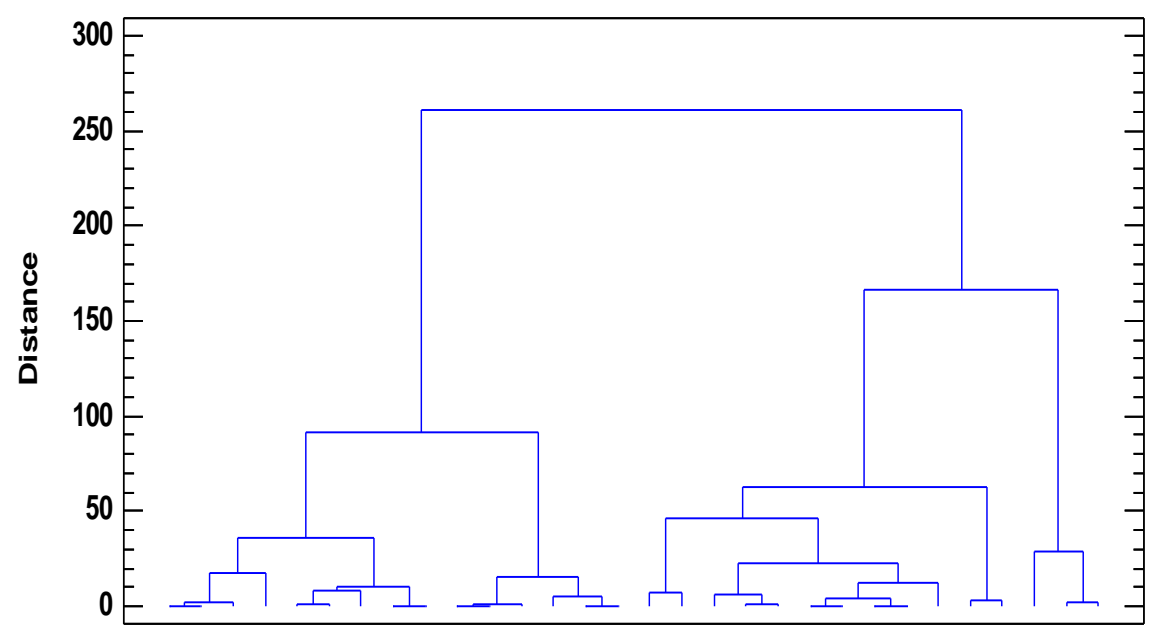

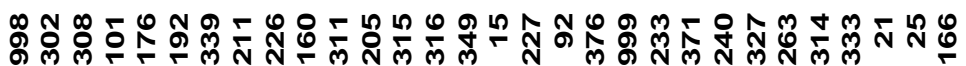

Figure 2. Dendrogram of Genotypes for Indices

Table 7. Eigen Values of the Correlation Matrix for Traits Using Stress and Non-Stress Condition's Mean

\begin{tabular}{ccccc}
\hline & $\begin{array}{c}\text { Eigen } \\
\text { value }\end{array}$ & Difference & $\begin{array}{c}\text { Proportion } \\
\text { of variance }\end{array}$ & $\begin{array}{c}\text { Cumulative } \\
(\%)\end{array}$ \\
\hline 1 & 4.101 & 1.730 & 0.512 & 51.26 \\
2 & 2.371 & & 0.296 & 80.90 \\
\hline
\end{tabular}

Table 8. Component Matrix for Traits Using Stress and Non-Stress Condition's Mean

\begin{tabular}{ccc}
\hline & Factor & Factor \\
& 1 & 2 \\
\hline Flowering & $\mathbf{- 0 . 7 3 9}$ & $\mathbf{0 . 6 3 2}$ \\
Podding & $\mathbf{- 0 . 7 0 0}$ & $\mathbf{0 . 6 5 2}$ \\
Maturity & -0.273 & $\mathbf{0 . 8 3 1}$ \\
Total dry & $\mathbf{0 . 7 9 5}$ & 0.491 \\
matter & & \\
Seed number & $\mathbf{0 . 6 0 3}$ & $\mathbf{0 . 6 3 7}$ \\
Yield & $\mathbf{0 . 8 8 1}$ & 0.446 \\
100-seed & $\mathbf{0 . 8 0 9}$ & -0.078 \\
weight & & \\
Harvest & $\mathbf{0 . 7 5 1}$ & 0.063 \\
index & & \\
\hline
\end{tabular}

Extraction method: Principal Component Analysis

Table 9. Eigen Values of the Correlation Matrix for Indices

\begin{tabular}{ccccc}
\hline & $\begin{array}{c}\text { Eigen } \\
\text { value }\end{array}$ & Difference & $\begin{array}{c}\text { Proportion } \\
\text { of variance }\end{array}$ & $\begin{array}{c}\text { Cumulative } \\
(\%)\end{array}$ \\
\hline 1 & 4.835 & 0.810 & 0.537 & 53.72 \\
2 & 4.025 & & 0.447 & 98.44 \\
\hline
\end{tabular}


Table 10. Component Matrix Based on Indices

\begin{tabular}{ccc}
\hline & Factor & Factor \\
& 1 & 2 \\
\hline Yp & $\mathbf{0 . 9 6 4}$ & -0.244 \\
Ys & $\mathbf{0 . 6 4 7}$ & $\mathbf{0 . 7 5 8}$ \\
MP & $\mathbf{0 . 9 9 1}$ & 0.125 \\
TOL & $\mathbf{0 . 6 7 7}$ & $\mathbf{- 0 . 7 1 8}$ \\
GMP & $\mathbf{0 . 9 9 2}$ & 0.100 \\
STI & -0.345 & $\mathbf{0 . 9 2 4}$ \\
HARM & $\mathbf{0 . 8 7 5}$ & 0.474 \\
RDI & -0.345 & $\mathbf{0 . 9 2 4}$ \\
DI & 0.242 & $\mathbf{0 . 9 5 8}$ \\
\hline
\end{tabular}

Extraction method: Principal Component Analysis

\subsection{Biplot Analysis}

\subsection{Characters}

The biplot analysis based on PCA for stress and non-stress conditions mean shown in Figure 3. According to this figure, genotypes 21, 101, 308, and 302 showed maximum values of NS, TDM, and YI traits. However, the greatest amount of days to flowering, podding, and maturity seen in genotypes $211,316,15$, and 205 . In addition, the highest of SW and HI values observed in cultivar 998 and genotypes 176, 166, and 25.

\subsubsection{Indices}

The results of biplot for genotypes based on tolerance indices showed that the most values for STI and RDI belonged to genotype 227. Genotypes 15, 25, and 166 have highest amount of DI index. Genotype 21 with maximum amount of Ys, HARM, GMP, and MP distinguished from the rest of genotypes. Finally, the greatest values of Yp and TOL indices seen for genotypes 101, 302, 308, 176, 192, 226, and 211 and cultivar 998 (Figure 4).

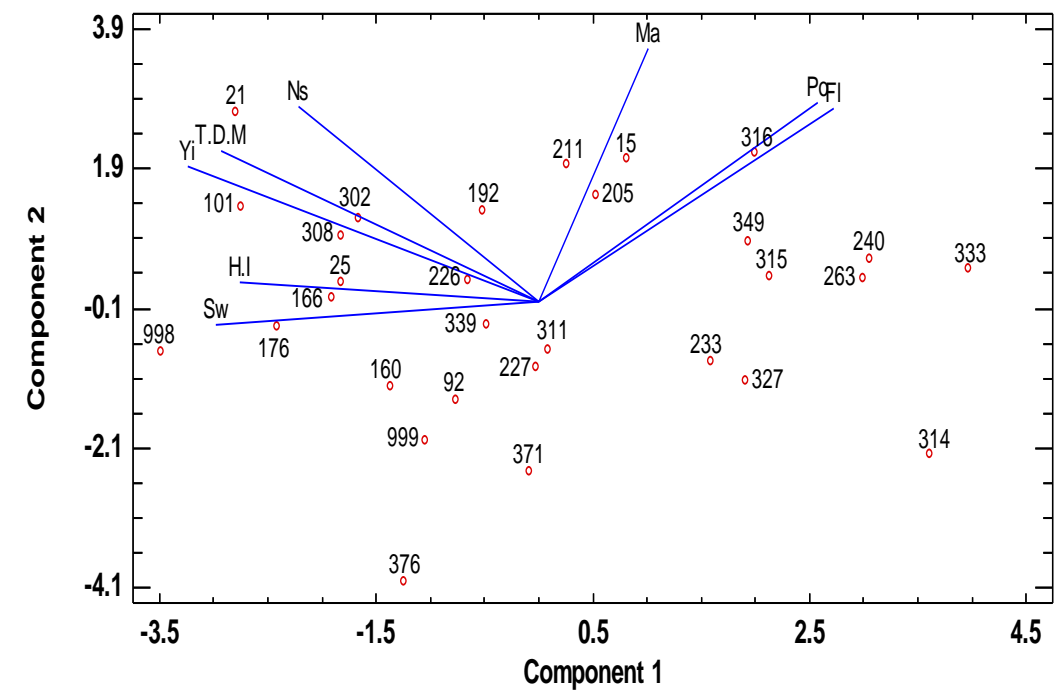

Figure 3. Biplot Analysis of Traits Using Components 1 and 2 for Stress and Non-Stress Condition's Mean 


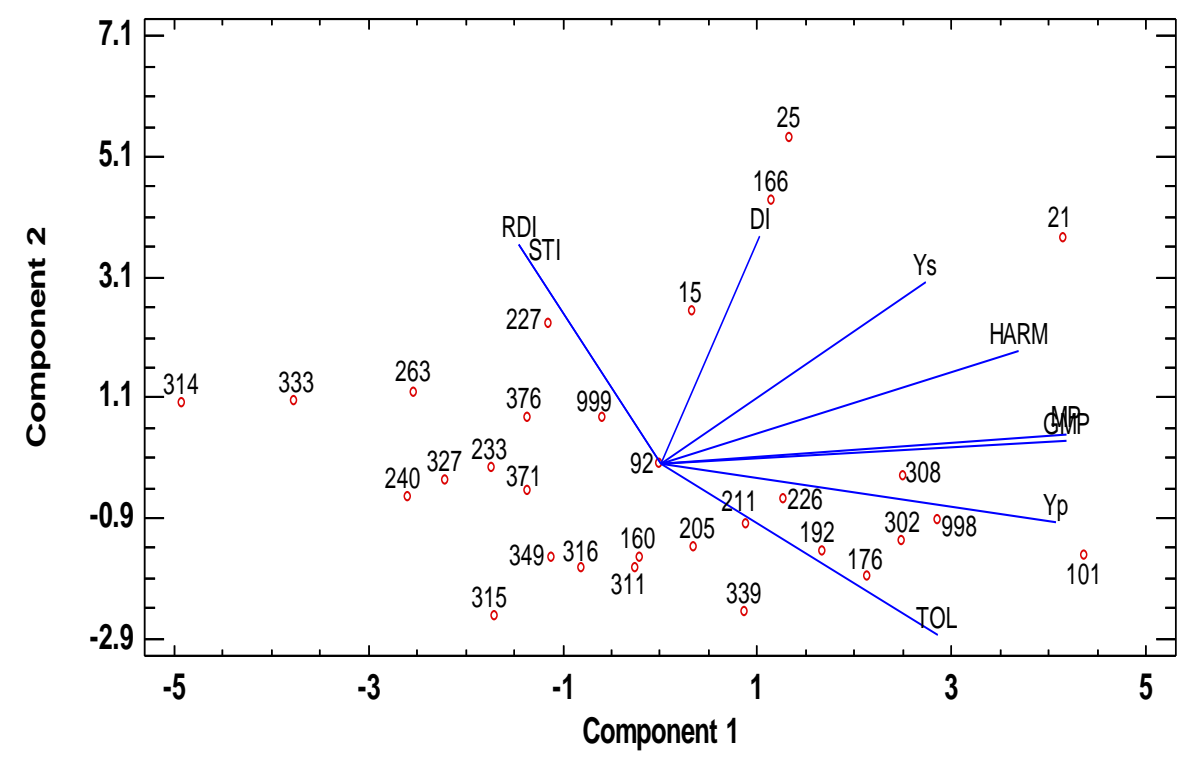

Figure 4. Biplot Analysis for Indices Using Components 1 and 2

\section{Discussion}

Rarity of water is a main restraint limiting grain legumes production predominantly in the arid and semi-arid tropics [15]. The genetically discovery of variable accessions is the basic source to conservation of germplasm and potential breeding materials for the future [16]. The further development of accessions level data and subsequent comparison those of at the across collections would greatly facilitate identification of unique accessions [16]. Drought impacts affect most of plant life aspects such as decrease performance, growth, and resistance to other environmental stresses [17]. Drought stress causes pollen grain sterility [18], which reduce the plants performance. A strongly negative correlation detected at the flowering and pooding times with grain yield and its components relationships; However, maturity time was a phenology character that has not a strong association with ones of the crop (Table 8). Hence, it could be said chickpea make a compensatory gap between start of reproductive phase and its end to alleviation adversity of terminal drought as well as flowering period.

Drought-induced reduction in the time for grain filling resulted in smaller grains in chickpea [19]. Farooq et al. [15] mentioned some of major traits related to chickpea grain yiled in drought stress conditions, so that duration of growth had not correlation with that [20], while grain size, early maturity, and plant height have significant correlation with this character [21]. Traits such as early flowering, podding and maturity provide an escape mechanism, and may be used for mass screening [22]. Grain yield and seed number decreased by drought, so that more performance genotypes have high seed numbers, too. The genotype 21 has highest grain yield and seed number, furthermore showed highest Ys, MP, GMP, and HARM indexes, which shown this genotype could be has a acceptable performance for relatively drought regions. The most Yp and TOL values revealed for genotype 101 which has highly seed number and 100-seed weight that these results make it as a interested genotypes for irrigated farming systems. Yp, MP, GMP, and HARM were indices that genotype 314 which has low amount of them and showed lowest grain yield, seed number and 100-seed weight than rest genotypes. Screening and mass selection may be useful to obtain desirable phenotypic characteristics based on the traits strongly correlated with yield [14]. Our observations did not show any correlation between phenology traits and yield, more staying green, although, helped to more moderately remobalization of stored materials to grain. Grain yield has not correlation 
with seed weight, alternatively total dry matter showed greatest with that (Tables 4,5 , and $6)$.

Those genotypes that have high values for drought resistance index (DI), which has most value in second factror (Table 10), have been known as elit lines in the this work. The genotypes 21, 25, and 166 detected as moer tolerant in the average of drought stress and non-stress conditions. The susceptible genotypes also were 314 and 333 that can were useful bases as extreme selected lines for mechanisms studies in development breeding programs to drought tolerance in chickpea.

\section{Acknowledgments}

We acknowledge The Iranian Pulse Project Excellence for supporting this work.

\section{References}

[1] FAO, FAO STAT Data, "Food and Agriculture Organization of the United Nations", (2012), http://Faostat.Fao.Org/.

[2] S. H. Sabaghpour, A. A. Mahmodi, A. Saeed, M. Kamel, and R. S. Malhotra, "Study on Chickpea Drought Tolerance Lines Under Dryland Condition Of Iran”, Indian Journal of Crop Science, vol. 1, no. 1-2, (2006), pp. 70-73.

[3] A. Taleei and J. shaabani, "Yield potential Analysis of Desi Chickpea Genotypes in Water Stress Conditions", Advanced Science and Technology Letters, vol. 142, (2016), pp. 9-16.

[4] F. Nawaz, R. Ahmad, E. A. Waraich, M. S. Naeem, and R. N. Shabbir, "Nutrient Uptake, Physiological Responses, and Yield Attributes of Wheat (Triticum Aestivum L.) Exposed to Early and Late Drought Stress", Journal of Plant Nutrition, vol. 35, no. 6, (2012), pp. 961-974.

[5] P. Ramirez-Vallejo, and J. D. Kelly, "Traits Related to Drought Resistance in Common Bean", Euphytica, vol. 99, no. 2, (1998), pp. 127-136.

[6] R. A. Richards, G. J. Rebetzke, A. G. Condon and A. F. V. Herwaarden, "Breeding Opportunities for Increasing the Efficiency of Water Use and Crop Yield in Temperate Cereals", Crop Science, vol. 42, no. 1, (2002), pp. 111-121.

[7] S. S. Mardeh, A. Ahmadi, K. Poustini, and V. Mohammadi, "Evaluation of Drought Resistance Indices under Various Environmental Conditions", Field Crops Research, vol. 98, no. 2, (2006), pp. 222-229.

[8] J. M. Clarke, R. M. DePauw, and T. F. Townley-Smith, "Townley-Smith, Evaluation of Methods for Quantification of Drought Tolerance in Wheat", Crop Science, vol. 32, no. 3, (1992), pp. 723-728.

[9] Hall, "Is Dehydration Tolerance Relevant to Genotypic Difference in Leaf Senescence and Crop Adaption to Dry Environments?", Current Topics in Plant Physiology, USA, (1993).

[10] R. A. Fischer, D. Rees, K. D. Sayre, Z. M. Lu, A. G. Condon, and A. L. Saavedra, "Wheat Yield Progress Associated with Higher Stomatal Conductance and Photosynthetic Rate, and Cooler Canopies", Crop Science, vol. 38, no. 6, (1998), pp. 1467-1475.

[11] L. Jusheng, "Comparison of Evaluating Methods for Agronomic Drought Resistance in Crops", Acta Agriculturae Boreali-Occidentalis Sinica, vol. 3, (1998).

[12] Rosielle, and J. Hamblin, "Theoretical Aspects of Selection for Yield in Stress and Non-Stress Environment”, Crop Science, vol. 21, no. 6, (1981), pp. 943-946.

[13] M. Cordovilla, F. Ligero, and C. Lluch, "Growth and Nitrogen Assimilation in Nodules in Response to Nitrate Levels in Vicia Faba under Salt Stress", Journal of Experimental Botany, vol. 47, no. 2, (1996), pp. 203-210.

[14] G. C. Fernandez, "Effective Selection Criteria for Assessing Plant Stress Tolerance", In Proceedings of the International Symposium on Adaptation of Vegetables and other Food Crops in Temperature and Water Stress, (1992).

[15] M. Farooq, N. Gogoi, S. Barthakur, B. Baroowa, N. Bharadwaj, S. S. Alghamdi, and K. H. M. Siddique, "Drought Stress in Grain Legumes during Reproduction And Grain Filling", Journal of Agronomy and Crop Science, (2016).

[16] Z. Ahmad, A. S. Mumtaz, M. Nisar, and N. Khan, "Diversity Analysis of Chickpea (Cicer Arietinum L.) Germplasm and its Implications for Conservation and Crop Breeding", Agricultural Sciences, vol. 3, no. 5, (2012), p. 723.

[17] A. Taleei and J. shaabani, "Yield potential Analysis of Kabuli Chickpea Genotypes in Water Stress Conditions", Advanced Science and Technology Letters, vol. 142, (2016), pp. 1-8.

[18] G. Duc, H. Agrama, S. Bao, J. Berger, V. Bourion, A. M. De Ron, and A. Tullu, "Breeding Annual Grain Legumes for Sustainable Agriculture: New Methods to Approach Complex Traits and Target New Cultivar Ideotypes”, Critical Reviews in Plant Sciences, vol. 34, no. 1-3, (2015), pp. 381-411.

[19] A. Al-Ghzawi, S. Zaitoun, H. Z. Gosheh, and A. M. Alqudah, "The Impacts of Drought Stress on Bee Attractively and Flower Pollination of Trigonella Moabitica (Fabaceae)", Arch Agron Soil Sciences,. vol. 55, no. 6, (2009), pp. 683-692. 
[20] S. L. Davies, N. C. Turner, K. H. M. Siddique, L. Leport, and J. A. Plummer, "Seed Growth of Desi and Kabuli Chickpea (Cicer Arietinum L.) in a Short-Season Mediterranean-Type Environment”, Animal Production Science, vol. 39, no. 2, (1999), pp. 181-188.

[21] J. Kashiwagi, L. Krishnamurthy, J. H. Crouch, and R. Serraj, "Variability of Root Length Density and its Contributions to Seed Yield in Chickpea (Cicer Arietinum L.) under Terminal Drought Stress", Field Crops Research, vol. 95, no. 25, (2006), pp. 171-181.

[22] K. B. Singh, G. Bejiga, M. C. Saxena, and M. Singh, "Transferability of Chickpea Selection Indices from Normal to Drought-Prone Growing Conditions in a Mediterranean Environment", Journal of Agronomy and Crop Science, vol. 175, no. 1, (1995), pp. 57-63. 
International Journal of Bio-Science and Bio-Technology Vol.9, No.2 (2017) 